\title{
Impact of COVID-19 on postgraduate education and mental wellbeing of surgical trainees: a systematic review
}

\author{
Oshan Basnayake, Umesh Jayarajah, Kawmadi Gunawardena, Dharmabandhu N Samarasekera \\ Department of Surgery, Faculty of Medicine, University of Colombo, Sri Lanka
}

Keywords: COVID-19; SARS-CoV-2; postgraduate surgical training; trainees' perspective; mental wellbeing; postgraduate education; systematic review

\begin{abstract}
Introduction

Coronavirus disease COVID-19 is a global pandemic that has produced adverse effects on many facets of the health care system including surgical specialities. In this systematic review, we aimed to comprehensively analyze the global literature on the impact of COVID-19 on surgical education and the mental wellbeing of surgical trainees.
\end{abstract}

\section{Methods}

We searched PubMed, Scopus, LILACS, Ovid MEDLINE, Web of Science, Google Scholar and Cochrane CENTRAL between 1-January 2020 and 20-January 2021 and performed a systematic review of all observational and experimental studies on the impact of COVID-19 on postgraduate surgical trainees of all specialities. Operative and clinical exposure, surgical education and mental wellbeing were assessed as outcome measures.

\section{Results}

Fifty-nine studies including 11544 surgical trainees were analyzed. The majority of the trainees were general surgical trainees $[n=4435,38.4 \%]$. Most studies $[n=40,67.8 \%]$ were conducted at national level in high income countries $[n=9480$; $82.1 \%$ ]. Most studies reported a significant reduction in the operative exposure [range 45.7-100\%] including elective, emergency and other clinical encounters. The mental health of trainees was affected [11.3-100\%] and depression [10.6$40.8 \%$, anxiety [31.7-69\%] and burnout [7.1-68\%] were noticed. Concerns on contraction and transmission of COVID-19 infection and delays in surgical training and career progression were also reported.

\section{Conclusion}

COVID 19 pandemic had caused a considerable impact on surgical training, teaching, learning activities and mental

\footnotetext{
Correspondence: Dharmabandhu N Samarasekera

E-mail: samarasekera58@yahoo.co.uk

(iDhttps://orcid.org/0000-0003-2229-7549

Received: 07-09-2021 Accepted: 22-11-2021 DOI: http://doi.org/10.4038/sljs.v39i3.8888
}

well-being of the trainees. Measures should be taken to mitigate these effects to improve surgical training in the era of the COVID pandemic.

\section{Introduction}

On 11th March 2020 Coronavirus disease COVID-19 was declared a global pandemic by the World Health Organization [1]. As of September 2021, greater than 200 million cases and over 4.5 million deaths have been reported globally [2]. The COVID-19 pandemic has produced significant effects on various aspects of human life including the global economy $[3,4]$. Furthermore, a considerable impact on various types of health care and services including the surgical subspecialities has been noted [5-7]. With the current rising trend in the incidence of COVID-19 and lack of results on the long term efficacy of vaccines, future implications remain uncertain [8].

Both undergraduate and postgraduate training programs were also affected during the COVID-19 pandemic. The impact on surgical training was more pronounced. In surgical training, the development of technical and hands-on skills is a mandatory component among many other aspects. Several aspects such as reduction in clinical and operative exposure, cancellation of professional courses and exams and reduction of foreign training and prospective fellowship opportunities have caused major concerns in relation to surgical training [9, 10]. Several studies have attempted to describe the impact of COVID-19 on postgraduate education and the mental wellbeing of surgical trainees. However, to date, an objective assessment and systematic review of published literature have not been performed. A timely review is necessary to objectively assess the existing literature which would be useful for future recommendations. Therefore, we aim to perform a systematic review of published global literature in relation to the impact of COVID-19 and surgical education and the mental wellbeing of surgical trainees.

\section{Methods}

A systematic review of the literature was performed including all observational and experimental studies on the impact of COVID-19 on postgraduate education and mental wellbeing of surgical trainees of all specialities. The systematic review was performed in accordance with the PRISMA guidelines. 
Figure 1. PRISMA flow chart

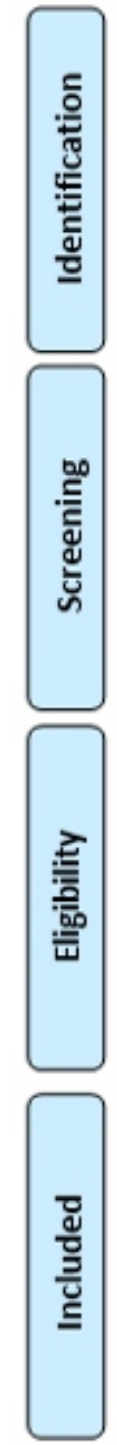

The primary objective of this systematic review was to perform a comprehensive analysis on the impact on the postgraduate education of surgical trainees of all specialities.

\section{Search strategy}

Articles published between 1 January 2020 and 20 January 2021 in PubMed, Scopus, LILACS, Ovid MEDLINE, Web of Science, Google Scholar and Cochrane CENTRAL were searched using specified search terms in the title or abstract fields. The detailed search strategy is provided in the supplementary file. Articles published in all languages and all surgical subspecialties globally were included.

Articles that mentioned both subjective and objective assessments of the impact of COVID-19 on surgical training were included in the analysis. The reference lists of all selected full papers were also assessed to identify additional papers for the systematic review.
All articles were screened for eligibility by two independent investigators based on the titles, abstracts and keywords of citations from the electronic databases. Full texts of all selected records were assessed for eligibility. All studies describing the clinical and operative exposure, surgical education / continuous professional development activities and mental wellbeing among trainees of all surgical specialities were included. Opinions/ perspectives were excluded from the analysis. Eligible studies were determined by consensus between two investigators. In doubtful instances, the opinion of senior investigators was sought.

Data extracted from individual studies were organized into three main categories;

1] Operative and clinical exposure

2] Surgical education and continuous professional development

3] Psychological aspects and mental wellbeing 
Table 1. Percentage distribution of trainees from various subspecialties

\begin{tabular}{|l|r|r|}
\hline Surgical specialty & N & \% \\
\hline General & 4435 & 38.4 \\
\hline Ophthalmology & 1650 & 14.3 \\
\hline Orthopedic & 1077 & 9.3 \\
\hline Neurosurgery & 1036 & 9 \\
\hline Urology & 975 & 8.4 \\
\hline Otolaryngology & 903 & 7.8 \\
\hline Plastic & 344 & 3 \\
\hline Other & 1124 & 9.7 \\
\hline Total & 11544 & 100 \\
\hline
\end{tabular}

Finally, the available data were tabulated and assessed qualitatively. A meta-analysis could not be performed due to considerable variations in the study methodology, assessment tools and reporting [Table-S1].

The quality of the included cross-sectional studies was assessed using Joanna Briggs Institute [JBI] critical appraisal checklist for cross-sectional studies. Each study was graded based on the cumulative points and percentage [Poor $<50 \%$, Fair 50-75\%, Good $>75 \%$ ].

\section{Results}

\section{Characteristics of included studies}

A total of 2135 studies were identified after the initial search. The process of selection of studies and elimination is summarized in the PRISMA flow chart [Figure 1]. After excluding the duplicates and non-relevant studies during the initial screening, a total of 127 full texts were assessed for eligibility. Finally, 59 studies describing responses of 11544 surgical trainees were included in the study. The majority of trainees were general surgical trainees $[n=4435,38.4 \%]$ followed by ophthalmology [14.3\%], orthopaedic [9.3\%] and neurosurgery [9\%] [Table 1$]$.

Studies were conducted among trainees from different countries [low and high income] and settings and at different levels of training. Most studies [ $n=40,77.6 \%$ of total sample size] were conducted at the national level, 8 [13\%] among trainees from multiple countries, 5 [7.2\%] at multiple centres within a single country and $6[2.1 \%]$ at single centres. The response rate was mentioned in 23 studies with an average response rate of $52.4 \%$ [range 12.2-100\%]. Most of the studies $[n=49,83 \%$ ] used a non-validated subjective questionnaire for the assessment of the impact on surgical training. The number of participants who had been infected with COVID-19 at the time of the survey was mentioned in 10 studies with a total number of 98 out of 1963 infected trainees. The prevalence of COVID -19 in this subgroup was 5\% [range 2.2-8\%]. The quality of the included cross-sectional studies are shown in table $\mathrm{S} 1$ and the majority were either fair $[30.5 \%, 18]$ or poor $[69.5 \%, 41]$.

The majority were conducted in high income countries $[\mathrm{n}=9480,82.1 \%]$ followed by low-middle income countries $[\mathrm{n}=1734,15 \%]$ and upper middle income countries $[\mathrm{n}=330$, $2.9 \%$.

\section{Impact of COVID-19 on operative and clinical exposure} A total of $51[n=10270$ trainees, 89\%] studies assessed the impact of COVID-19 on operative and clinical exposure [Table S2-supplementary file]. Five studies $[n=602,5.9 \%$ ] used pre-tested questionnaires [11-16]. Only 3 studies $[1.8 \%$, $\mathrm{n}=180$ trainees] performed an objective assessment of the reduction in the number of cases compared to pre-pandemic data by means of hospital and trainee records [Table S2]. Most studies reported a significant reduction in the operative exposure [range: 45.7-100\%]. Reduction in urgent/ emergency surgeries and trauma surgeries were mentioned in four $[n=1061,10.3 \%]$ and 2 studies $[n=234,2.3 \%$ ] respectively [range: $50-80 \%$ ] [Table S2]. A national-level study conducted in Ireland, which assessed the number of the orthopaedic operative procedure using trainees logbook records showed no reduction in nine specified trauma procedures in 2020 compared with 2018 and 2019 [17]. Data regarding laparoscopic and endoscopic procedures were reported only in 2 studies and both showed a considerable reduction $[18,19]$. Out of 51 studies, 39 studies $[\mathrm{n}=8587$, $83.6 \%$ ] reported on the non-operative clinical exposure in surgical training. Fifteen studies $[n=3253,31.6 \%]$ reported a reduction in outpatient clinic encounters [range 41.1-83\%] [Table S2]. Interestingly, the utilization of outpatient encounters using telehealth facilities [range 25-99\%] was reported in 6 studies [ $n=681,6.6 \%$ ] [Table S2]. Apart from the above, reduction in on-call experience and ward-round based learning were reported in 6 studies [range 54.3-79\%] [Table S2].

\section{Impact on psychological aspects and mental wellbeing of surgical trainees}

Out of 59 studies, $46[\mathrm{n}=8786,76.1 \%]$ assessed the impact on mental health among surgical trainees [table S3supplementary file]. However, only seven studies [n=1933, $22 \%$ ] used at least one validated scoring tool in the assessment of anxiety [Generalized Anxiety Disorder Assessment [GAD-7]: $\mathrm{n}=1534,17.4 \%$ ], depression [Patient Health Questionnaire [PHQ-9]: $n=1492,17 \%$ ], burnout [Modified Maslach Burnout inventory score: $\mathrm{n}=223,2.5 \%$ ] and stress [Mini Z burnout survey: $n=349,4 \%$ ] [Table S3]. Four studies $[\mathrm{n}=379,4.3 \%]$ used pre-tested questionnaires [Table S3]. Out of 46, 17 studies [ $n=3797,43.2 \%$ ] assessed the anxiety, depression and stress among trainees [Table S3]. 
Except for one study, all have reported high levels of anxiety [31.7-69\%], depression [10.6-40.8\%] and stress [28-54.8\%] among trainees. However, Osama.et.al $[\mathrm{n}=112,1.3 \%]$ reported a reduction of Modified Maslach Burnout inventory score $[\mathrm{p}<0.001][20]$. Reduction in working hours among trainees may have contributed to this observation [12]. The majority of the trainees $[\mathrm{n}=5065,57.6 \%]$ reported concerns about contracting COVID-19 infection or transmitting the virus to family members or friends as the reason for the impact on mental health. In addition, reduction in surgical exposure /clinical training $[\mathrm{n}=5687,64.2 \%]$, inadequacy of personal protective equipment $[\mathrm{n}=3727,42.4 \%]$ and redeployment to other units $[\mathrm{n}=2357,26.8 \%]$ were reported as other reasons. Aziz et.al $[n=1102,12.5 \%]$ reported that although $65.1 \%$ of the respondents had 5 or more off-duty days compared to the pre-pandemic period, $33.1 \%$ reported more burnout and $72.7 \%$ were concerned about contracting or transmitting the virus to their family and friends [21]. Interestingly, Johnson et al. reported that $50.4 \%$ of respondents had no anxiety according to the GAD-7 score [GAD-7 score $<5][22]$.

\section{Impact on surgical education and continuous professional development}

Forty-one studies [ $\mathrm{n}=7971,69 \%$ ] assessed the impact of COVID-19 on surgical educational activities [Table S4supplementary file]. Thirty-three studies [ $\mathrm{n}=6427,80.6 \%$ ] reported an increase in the utilization of web-based teaching activities including webinars, online symposiums, lectures and videos of surgeries. Responders from 3 studies $[n=414$, $5.2 \%$ ] reported the usefulness and likelihood of continuing online educational activities after the pandemic [Table S4]. Chang et.al $[n=229,2.9 \%]$ reported that the average satisfaction regarding online-based teaching was lower compared to conventional teaching activities [23]. Nine studies $[\mathrm{n}=731 ; 9.1 \%]$ reported an increase in research activities and projects among surgical trainees due to reduced clinical workload [Table S4]. Payne. et.al evaluated the impact of redeployment of responders to ICU and reported that $97 \%[n=32]$ reported that the redeployment had a positive impact on gaining knowledge and confidence in managing critically ill patients [24]. Bandi et al. has reported the usefulness of utilizing a dissection laboratory for Otolaryngology trainees [25].

\section{Discussion}

Although the direct impact of the COVID-19 pandemic on the health of the public in terms of morbidity and mortality is widely known, there are subtle ramifications due to the pandemic which are not clearly revealed. One such consequence is the impact on postgraduate medical training, especially in the surgical specialities $[16,17]$. This is because surgical training includes both clinical patient care and also accumulation of technical and operative skills. Most components of surgical training have been affected due to the delays in acquiring adequate training and increased psychological stress [26].

In this systematic review, the impact on surgical training was considerable in all three aspects analyzed such as operative and clinical training, surgical educational activities and mental wellbeing. The overall operative exposure was reduced by $45.7-100 \%$, while urgent/ emergency surgeries and trauma surgeries were reduced by $50-80 \%$ [Table S2]. Furthermore, studies have also shown a considerable reduction in laparoscopic and endoscopic procedures, outpatient clinic encounters, on-call experience and wardround based learning. Measures taken to control the disease spread such as social distancing and lockdown have led to cancellation or delay of elective operations and minimizing patient visits at outpatient clinics. Restriction of routine clinical activities protects health care workers and patients from the viral transmission. Furthermore, personal protective equipment can be prioritized and preserved for the care of COVID-19 positive patients who need mandatory treatment. Moreover, important resources such as hospital beds and intensive/ critical care beds may be reserved for symptomatic and critically ill COVID-19 patients.

Several studies reported a reduction or delay of postgraduate educational or continuous professional development programs [Table S4]. However, as a measure to overcome this problem, increased utilization of online teaching activities such as online symposia, webinars, lectures and videos of surgeries were conducted. Although several studies mentioned the usefulness and likelihood of continuing such activities after the pandemic, few studies reported that the average satisfaction of online-based teaching was lower compared to conventional teaching methods [Table S4].

The reduction in clinical activities and operative exposure has a potential negative impact on postgraduate surgical training as the acquisition of clinical expertise and surgical skills are greatly dependent on the level of clinical exposure and handson operative experience. Delay and postponement of such activities would negatively impact the career progression of surgical trainees. Due to the reduction of clinical workload, a considerable proportion of surgical trainees may not be able to fulfil their criteria in terms of clinical rotations and surgical exposure leading to delays in accreditation and qualifications for memberships or fellowships of postgraduate surgical colleges.

The physical and mental well-being of postgraduate surgical trainees were also negatively affected during the pandemic. Almost all studies that described the level of psychological wellbeing reported high levels of anxiety, depression and stress. However, a few studies reported a reduction of burnout 
compared to the pre-pandemic era which can be attributed to the reduction in working hours. Importantly, most trainees reported concerns about the inadequacy of personal protective equipment, redeployment to different non-surgical units, contracting COVID-19 infection and transmitting to family members and loved ones as considerable sources of stress. In this systematic review, we found 10 studies describing 98 out of 1963 infected trainees with a considerable prevalence of 5\%. Furthermore, delays in fulfilling criteria for accreditation due to reduction in surgical exposure /clinical training were additional concerns [Table S2].

Several positive aspects were also highlighted in these studies. Nine studies reported an increase in research activities and projects among surgical trainees due to reduced clinical workload [Table S4]. Online global platforms have enabled surgical trainees and surgeons to conduct worldwide collaborative researches on the surgical aspects of COVID-19 [27-29]. Web-based educational activities and conferences played an important role during the pandemic to enhance continuous professional development [30]. On a positive note, web-based activities are readily accessible with considerable cost-effectiveness for the organizers and the participants. Few studies evaluated the impact of redeployment of surgical trainees to ICU and reported that 97\% had a positive impact on gaining knowledge and confidence in managing critically ill patients.

\section{Limitations}

We identified several limitations in the published studies. The majority of studies did not utilize objectively validated assessment methods. There were considerable variations in the methodology and the reporting of findings precluding a meta-analysis. The majority of the studies were rated as either fair or poor. Although short term impact has been discussed, the long term implications seem unpredictable.

\section{Conclusion}

COVID 19 pandemic has caused a considerable impact on surgical training, career progression teaching-learning activities and mental well-being of the trainees. With the ongoing global pandemic and lack of data on the long term efficacy of vaccines or treatment to date, future directions of the pandemic remain uncertain. Therefore, implementing alternative options to mitigate the negative effects on surgical training should be actively considered.

Authors' contributions: Authors' contributions: $\mathrm{OB}, \mathrm{KG}$ and UJ designed the tables; collected, analyzed, and interpreted data; and wrote the article. $\mathrm{OB}, \mathrm{KG}$ and UJ formulated the concept and design of study, acquisition of data and analysis and drafting the article. DNS contributed to design and concept of study, revising it critically for important intellectual content and approval of the final version to be published. All authors have read and approved the final version of the manuscript.

All authors disclose no conflict of interest. The study was conducted in accordance with the ethical standards of the relevant institutional or national ethics committee and the Helsinki Declaration of 1975, as revised in 2000 .

\section{References}

1. Borges do Nascimento IJ, Cacic N, Abdulazeem HM, von Groote TC, Jayarajah U, Weerasekara I, et al. Novel coronavirus infection [COVID-19] in humans: a scoping review and metaanalysis. Journal of clinical medicine. 2020;9[4]:941. doi: $10.3390 /$ jcm 9040941

2. World Health Organization. Coronavirus disease [COVID-2019] situation reports. 2020.

https://www.who.int/emergencies/diseases/novel-coronavirus2019/situation-reports

3. Borges do Nascimento IJ, von Groote TC, O'Mathúna DP, Abdulazeem HM, Henderson C, Jayarajah U, et al. Clinical, laboratory and radiological characteristics and outcomes of novel coronavirus [SARS-CoV-2] infection in humans: A systematic review and series of meta-analyses. PloS one. 2020;15[9]:e0239235.

https://doi.org/10.1371/journal.pone.0239235

4. Jayasinghe R, Ranasinghe S, Jayarajah U, Seneviratne S. Quality of online information for the general public on COVID-19. Patient Education and Counseling. 2020;103[12]:2594-7. doi: 10.1016/j.pec.2020.08.001

5. Jayasinghe R, Jayarajah U, Seneviratne S. Consensus on perioperative surgical practice during the COVID-19 pandemic: an appraisal of the literature. Sri Lanka Journal of Surgery. 2020;38[2]:57-61.

http://doi.org/10.4038/sljs.v38i2.8738

6. Jayasinghe R, Welikala N, Gunaratne R, Jayarajah U, Dassanayake V, Seneviratne S. Anaesthetic implications during the COVID-19 pandemic: an appraisal of the literature. Sri Lanka Journal of Surgery. 2021;39[1]:48-52. http://doi.org/10.4038/sljs.v39i1.8814

7. Glasbey JC, Bhangu A, Collaborative C. Elective cancer surgery in COVID-19-free surgical pathways during the SARS-CoV-2 pandemic: an international, multicenter, comparative cohort study. Journal of Clinical Oncology. 2020:JCO. 20.01933. doi: $10.1200 / J C O .20 .01933$

8. Seneviratne SL, Jayarajah U, Abeysuriya V, Rahman A, Wanigasuriya K. COVID-19 Vaccine Landscape. Journal of the Ceylon College of Physicians. 2020;51:120-31. http://doi.org/10.4038/jccp.v51i2.7906

9. White EM, Shaughnessy MP, Esposito AC, Slade MD, Korah M, Yoo PS. Surgical Education in the Time of COVID: Understanding the Early Response of Surgical Training Programs to the Novel Coronavirus Pandemic. Journal of surgical education. 2021;78[2]:412-421 doi: 10.1016/j.jsurg.2020.07.036 
10.Cai Y, Jiam NT, Wai KC, Shuman EA, Roland LT, Chang JL. Otolaryngology Resident Practices and Perceptions in the Initial Phase of the US COVID-19 Pandemic. The Laryngoscope. 2020; 130[11]:2550-2557. doi: 10.1002/lary.28733

11.Collins C, Mahuron K, Bongiovanni T, Lancaster E, Sosa JA, Wick E. Stress and the Surgical Resident in the COVID-19 Pandemic. Journal of surgical education. 2021;78[2]:422-430. doi: 10.1016/j.jsurg.2020.07.031

12.Osama M, Zaheer F, Saeed H, Anees K, Jawed Q, Syed SH, et al. Impact of COVID-19 on surgical residency programs in Pakistan; A residents' perspective. Do programs need formal restructuring to adjust with the" new normal"? A cross-sectional survey study. International Journal of Surgery. 2020; 79:252-256. doi: 10.1016/j.ijsu.2020.06.004

13. Caruana EJ, Patel A, Kendall S, Rathinam S. Impact of Covid-19 on training and wellbeing in subspecialty surgery: a national survey of cardiothoracic trainees in the United Kingdom. The Journal of Thoracic and Cardiovascular Surgery. 2020; 160[4]:980-987.

doi: $10.1016 /$ j.jtcvs.2020.05.052

14.Paesano N, Santomil F, Tobia I. Impact of COVID-19 Pandemic on Ibero-American Urology Residents: Perspective of American Confederation of Urology [CAU]. International Brazilian Journal of Urology. 2020 Jul;46[suppl.1]:165-169. doi: 10.1590/S1677-5538.IBJU.2020.S120

15.Dash C, Venkataram T, Goyal N, Chaturvedi J, Raheja A, Singla $\mathrm{R}$, et al. Neurosurgery training in India during the COVID-19 pandemic: straight from the horse's mouth. Neurosurgical focus. 2020;49[6]:E16. doi: 10.3171/2020.9.FOCUS20537

16.Cox D, Wang P, Asch S, Mahmud E, Fearon WF, Silva N, et al. Impact of the COVID-19 Pandemic on Ophthalmology Residency Training in Portugal. Catheterization and cardiovascular interventions : official journal of the Society for Cardiac Angiography \& Interventions. 2020;33[10]:640-8. doi: 10.20344/amp. 14341

17.Sheridan GA, Hughes AJ, Quinlan JF. Quantifying the impact of the COVID-19 pandemic on orthopaedic trainees: a national perspective. Bone Joint Open. 2020 Oct 19;1[10]:645-652. doi: 10.1302/2633-1462.110.BJO-2020-0149.R1

18.Hennessy O, Fowler AL, Hennessy C, Hogan A, Nugent E, Joyce M. Covid 19 and Surgical training: Carpe Diem. The British journal of surgery. 2020;107[12]:e591. doi: 10.1002/bjs. 12032

19.Huamanchumo-Suyon ME, Urrunaga-Pastor D, Ruiz-Perez PJ, Rodrigo-Gallardo PK, Toro-Huamanchumo CJ. Impact of the COVID-19 pandemic on general surgery residency program in Peru: A cross-sectional study. Annals of medicine and surgery [2012]. 2020;60:130-4.

doi: 10.1016/j.amsu.2020.10.031
20.Osama M, Zaheer F, Saeed H, Anees K, Jawed Q, Syed SH, et al. Impact of COVID-19 on surgical residency programs in Pakistan; A residents' perspective. Do programs need formal restructuring to adjust with the "new normal"? A cross-sectional survey study. International Journal of Surgery. 2020;79:252-6. doi: $10.1016 / j$. .jsu.2020.06.004

21. Aziz H, James T, Remulla D, Sher L, Genyk Y, Sullivan ME, et al. Effect of COVID-19 on Surgical Training Across the United States: A National Survey of General Surgery Residents. Journal of surgical education. 2021;78[2]:431-439. doi: 10.1016/j.jsurg.2020.07.037

22.Johnson AP, Wohlauer MV, Mouawad NJ, Malgor RD, Coogan SM, Sheahan MG, et al. The Impact of the COVID-19 Pandemic on Vascular Surgery Trainees in the United States. Annals of vascular surgery. 2021;72:182-190.

doi: 10.1016/j.avsg.2020.09.045

23.Chang D-G, Park J-B, Baek GH, Kim HJ, Bosco A, Hey HWD, et al. The impact of COVID-19 pandemic on orthopaedic resident education: a nationwide survey study in South Korea. International orthopaedics. 2020;44[11]:2203-10. doi: 10.1007/s00264-020-04714-7

24.Payne A, Rahman R, Bullingham R, Vamadeva S, Alfa-Wali M. Redeployment of Surgical Trainees to Intensive Care During the COVID-19 Pandemic: Evaluation of the Impact on Training and Wellbeing. Journal of Surgical Education. 2021;78[3]:813-819. doi: 10.1016/j.jsurg.2020.09.009

25.Bandi F, Karligkiotis A, Mellia J, Gallo S, Turri-Zanoni M, Battaglia P, et al. Strategies to overcome limitations in Otolaryngology residency training during the COVID-19 pandemic. European archives of oto-rhino-laryngology. 2020; 277[12]:3503-3506. doi: 10.1007/s00405-020-06228-9

26.Vallée M, Kutchukian S, Pradère B, Verdier E, Durbant È, Ramlugun D, et al. Prospective and observational study of COVID-19's impact on mental health and training of young surgeons in France. The British Journal of Surgery. 2020; 107[11]:e486-e488. doi: 10.1002/bjs. 11947

27.Jayarajah U, Basnayake O. The need for trainee-led surgical research collaborative in Sri Lanka. Sri Lanka Journal of Surgery. 2021;39[1]:53-4. http://doi.org/10.4038/sljs.v39i1.8753

28.Collaborative C, Collaborative G. Timing of surgery following SARS-CoV-2 infection: an international prospective cohort study. Anaesthesia. 2021: https://doi.org/10.1111/anae.15458.

29. Collaborative C. Outcomes from elective colorectal cancer surgery during the SARS-CoV-2 pandemic. Colorectal Disease. 2020: https://doi.org/10.1111/codi.15431.

30.Wijerathne PK, Nanayakkara K, Basnayake O, Gunapala N, Jayarajah U. Impact of COVID-19 on postgraduate surgical training: the trainees' perspective. Sri Lanka Journal of Surgery. 2020;38[3]:53-5.

http://doi.org/10.4038/sljs.v38i3.8769 